\title{
Introduction to the Special Issue on DSM-IV and Acquired Brain
}

Injury

Within the European forensic arena, and in those of most of the rest of the world as well, the DSM-IV TR is widely used for psychiatric diagnostic purposes as well as for the diagnosis of the psychiatric consequences of Traumatic or Acquired Brain Injury (TBI).

Most official court medical examiners in Europe are general medical practitioners, who normally have no specific medical speciality. In spite of the fact that these forensic specialists are neither psychiatrists nor neuropsychologists, they must explore and assess all individuals with traumatic brain injury (TBI) who come within their jurisdiction. Likewise, it is the responsibility of these examiners to evaluate, not only patients with TBI, but also people presenting any kind of lesion, traumatic or not, which present sequelae of any type. These can include orthopaedic, internal and coronary lesions in addition to any other kind of physical and mental consequences of injury.

The official court medical examiner must evaluate and submit an official diagnostic report to the court. The court places a high emphasis on these reports given that they are considered to be the most objective and unbiased with regard to the parties involved in litigation.

Normally, the official medical examiner does a clinical exploration of the patient and studies the medical and neuropsychological reports submitted on the part of the injured party. These reports may proceed from the national health system, hospitals or from physicians or neuropsychologists in private practice.

When confronted with the need to make a diagnostic evaluation, and lacking the necessary specialization in psychiatry or psychology to correctly assess traumatic brain injury sequelae, they will normally turn to respected manuals and handbooks. The most commonly used criteria are those of the DSM IV TR and the ICD10.

The ensuing scenario is generally as follows: A physician with no formal and specific training in the matter, must sign a psychiatric or neuropsychological report that may prove to be decisive in the financial compensation and/or possibilities of rehabilitation for the injured party. In most cases, this official report bears the greatest weight in tipping the balance of a court decision unless the patient's attorney can demonstrate inconsistencies contained within the official report by submitting specialized neuropsychological and/or psychiatric reports.

Obviously, the possibility of malingering and deception is high and forensic medical examiners are quite cautious with regard to self-report on the part of the patients as well as in carrying out clinical explorations. They will generally attempt to adjust the results of their explorations to the DSM IV TR "objective" criteria. However, neither are the DSM IV TR criteria objective, nor is the forensic medical examiner totally qualified to shoulder this responsibility.

This situation is exacerbated by the fact that knowledge within the field of the neurobehavioral, neurocognitive and social consequences of acquired brain injury is as yet in a state of expansion and growth.

Because official examiner reports have such a tremendous impact on the life of both patients and their families, and because the possibility of receiving adequate neurorehabilitation often depends on these reports, the need for clear diagnostic criteria is paramount. In addition to this, the impact of these reports on insurance companies affects insurance rates, causing an even more far-reaching effect on society in general.

This issue analyses some of the most current topics in order to generate open scientific discussion on some of the most salient aspects related to brain injury and the neuropsychiatric, neuropsychological, and forensic diagnosis of people who have sustained a traumatic brain injury.

The possibility of malingered brain injury must be carefully taken into account by clinicians when exploring a person with TBI in the process of litigation. Dr. Bordini, Dr. Chaknis, Dr. Ekman-Turner and Dr. 
Perna present an overview of advances in the clinical and neuropsychological assessment of malingering, as well as differential diagnosis. The phenomenology of depression after brain injury is addressed by Dr. Starkstein and Dr. Lichinsky, analysing the overlap between the symptoms of depression and signs associated with neurological diseases. My article conveys doubts with regard to the accuracy, specificity and validity of the diagnosis using the criteria of the DSM IV TR of Dementia Due to Head Trauma. Dr. Patrick, Dr. Rice and Dr. Hostler point out the weaknesses and strengths of the DSM IV TR criteria for children with TBI. Finally, Dr. Parker shows the controversy concerning Post-concussional Disorder and Post-Traumatic Stress Disorder.

It is my hope that these analyses provoke more research in this area and help towards the creation of a qualified advocacy for change in the direction of highly accurate DSM IV TR criteria, which would clearly be a great benefit to all concerned parties and to society in general.

Also included in this issue are four unsolicited articles from Dr. Beis and colleagues, Dr. Jung and colleagues, Dr. Bergen and colleagues and Ms. Vaccaro and colleagues.

José León-Carrión, Ph.D.

Guest Editor

Facultad de Psicologia, Universidad de Sevilla

Avd. San Francisco Javier s/n

41018 Sevilla

Spain

Tel.: +34954557688

Fax: +34954374558

E-mail: leoncarrion@us.es 